\title{
L'élevage de l'agneau à l'herbe l. Effets de l'âge au sevrage sur les performances d'agneaux élevés à l'herbe puis engraissés en bergerie
}

\author{
Sophie PRACHE, A. BRELURUT, M. THERIEZ \\ avec la collaboration technique de J. LEROUX et $\mathrm{H}$. CASSAGNES \\ I.N.R.A., Laboratoire de la Production ovine \\ Centre de Recherches de Clermont-Ferrand-Theix \\ Saint-Genès-Champanelle, F 63122 Ceyrat
}

\section{Résumé}

Cette étude concerne la production d'agneaux à l'herbe dans le système d'élevage suivant : naissance et six premières semaines de vie en bergerie, pâturage sous la mère avec complément à volonté, puis finition en bergerie après sevrage. Son objectif est de déterminer l'intérêt du sevrage et l'âge auquel il doit éventuellement être réalisé.

Quatre traitements sont expérimentés : sevrage à 6,12 ou 16 semaines (groupes $1,2,3$ ) et traitement témoin non sevré. Après sevrage, chaque groupe est séparé en trois lots (1981) ou deux lots (1982) d'engraissement, selon la vitesse de croissance avant sevrage.

La quantité d'herbe offerte au pâturage est élevée : 6,9 $\mathrm{kg}$ MS par brebis suitée et par jour, en moyenne sur la période expérimentale.

Même avec ces conditions très favorables, la vitesse de croissance des agneaux au pâturage est très liée à leur vitesse de croissance durant les six premières semaines de vie. Par contre, la vitesse de croissance augmente après le sevrage, et ne dépend plus de la vitesse de croissance avant celui-ci.

La consommation la plus faible d'aliment concentré est obtenue avec un sevrage réalisé à 16 semaines pour les agneaux à vitesse de croissance rapide sous la mère et à 12 semaines pour les agneaux à vitesse de croissance lente.

L'ensemble des résultats permet de conclure qu'un agneau à vitesse de croissance lente sous la mère doit être sevré précocement, alors qu'un agneau à vitesse de croissance plus rapide peut être laissé plus longtemps sous la mère.

Cette technique permet de réduire la consommation de concentré et l'âge à l'abattage des agneaux élevés à l'herbe.

Mots clés : Age au sevrage, sevrage, agneau d'herbe, agneau.

\section{Introduction}

En France, 60 p. 100 des agneaux sont élevés à l'herbe. Ils sont produits essentiellement en plein air intégral dans des régions à dominante herbagère ou én semi-plein air dans des régions de polyculture élevage. Les difficultés de ce type 
d'élevage (faibles croissances et problèmes de finition des agneaux, parasitisme) se sont accentuées lorsque les éleveurs ont intensifié leur production.

En effet, l'augmentation de la prolificité, d'une part (VILlette \& Theriez, 1981), et du chargement d'autre part (Jones \& SANLAND, 1974 ; Young \& Newton, 1975 ; Gibb \& Treacher, 1980) s'accompagnent généralement d'une diminution de la vitesse de croissance des jeunes.

Penning \& Gibb (1979) ont montré, avec des agneaux allaités artificiellement au pâturage, que la vitesse de croissance est positivement liée à la quantité de lait ingérée, même si l'herbe n'est pas limitante; les agneaux qui disposent de peu de lait tentent de compenser en ingérant plus d'herbe, mais cette compensation est limitée quantitativement (compte tenu de la différence de valeur énergétique entre l'herbe et le lait), et dans le temps (à partir de l'âge de 2,5 mois, ce sont les agneaux allaités à haut niveau de lait qui ingèrent le plus d'herbe).

Ces phénomènes sont encore accentués lorsque l'herbe n'est plus offerte à volonté. Gibi \& Treacher (1980) travaillant avec des brebis allaitantes conduites à 2 niveaux de chargement, ont montré qu'à fort chargement, non seulement la production laitière des mères, mais aussi l'ingestion d'herbe par les agneaux, étaient plus faibles qu'à faible chargement.

Ceci conduit à des problèmes d'élevage des jeunes et retarde leur finition au cours de l'été alors que l'herbe diminue en quantité et en qualité et que les risques d'infestation parasitaire augmentent.

La complémentation au pâturage et le sevrage, éventuellement suivi d'une finition en bergerie, permettent de limiter ces difficultés.

Cependant, il existe peu de données sur les quantités de concentré consommées par les animaux dans ce type de conduite. Nous avons regroupé, au tableau 1, des données obtenues par différents auteurs ; ces résultats montrent une grande variabilité dans les quantités de concentré consommées par les animaux, aussi bien pour ceux qui sont finis sous la mère au pâturage, que pour ceux qui sont sevrés à différents âges et finis soit à l'herbe, soit en bergerie.

En outre, l'amélioration de la prolificité conduit souvent à une augmentation de l'état d'engraissement à poids vif donné (THERIEz, 1984) et donc à des difficultés d'adaptation au marché qui s'oriente plutôt vers des carcasses lourdes et maigres. De nombreux auteurs s'accordent pour dire que le sevrage provoquerait une modification de la composition corporelle de l'animal résultant soit d'une mobilisation des réserves lipidiques (Mrtchell \& Jagush, 1972; Searle \& Griffiths, 1976), soit d'une chute brutale dans l'accumulation des graisses corporelles (Robelin et al., 1977 ; SeArle \& Griffiths, 1976). Le sevrage des agneaux pourrait donc aussi permettre de limiter l'état d'engraissement des carcasses.

Cette étude concerne la production d'agneaux de boucherie à l'herbe dans le système d'élevage suivant : naissance et six premières semaines de vie en bergerie, pâturage sous la mère avec complément à volonté, puis finition en bergerie après sevrage. Son objectif est de déterminer l'intérêt du sevrage et l'âge auquel il doit éventuellement être réalisé, de manière à optimiser la combinaison des paramètres suivants : croissance des agneaux et âge à l'abattage, quantités de concentré ingérées et qualité des carcasses. 


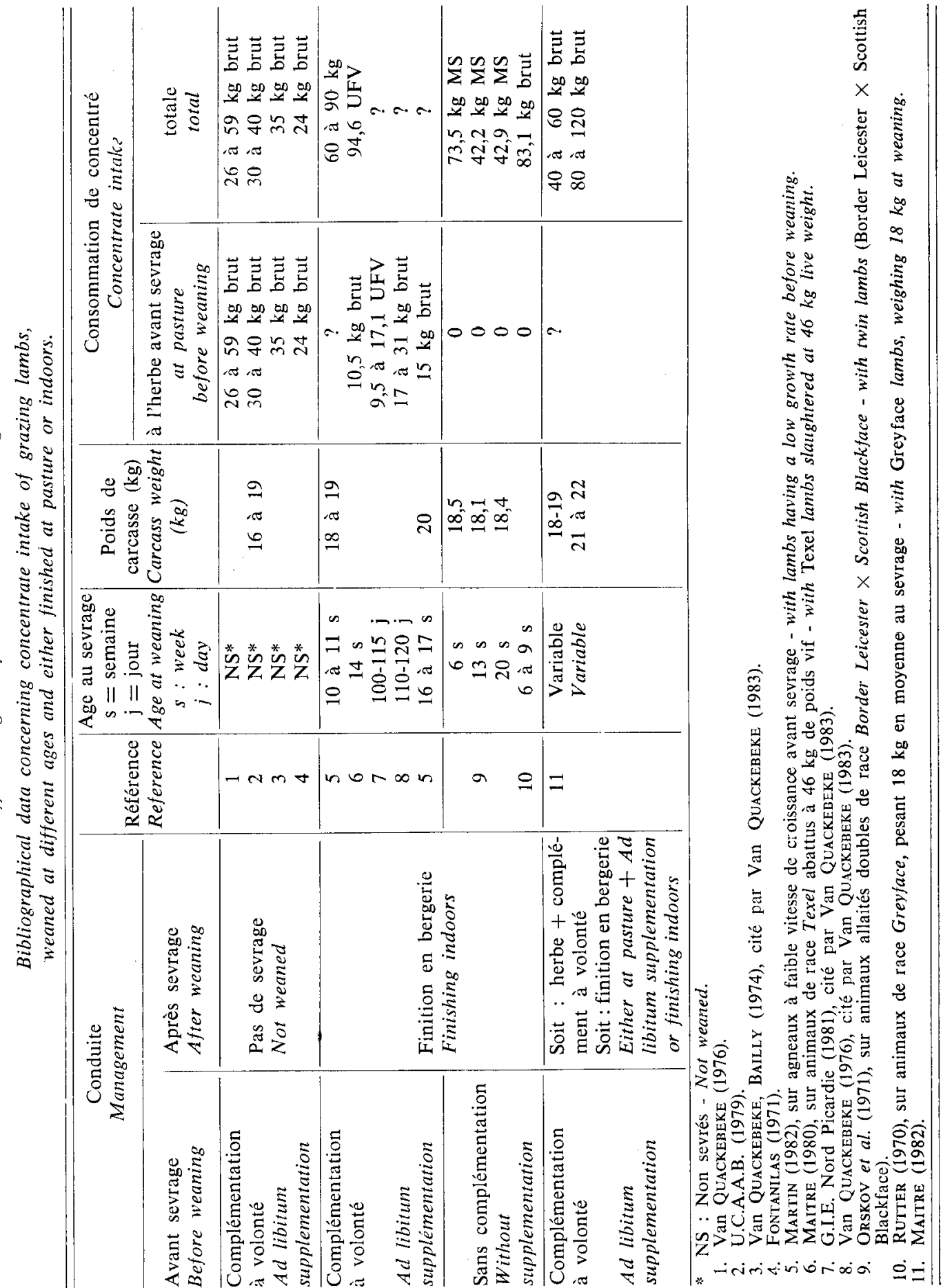

密

总

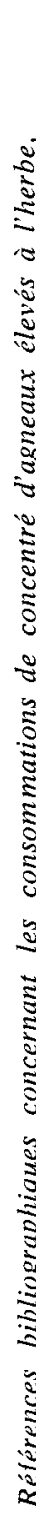


Plus globalement cet âge au sevrage conditionnera aussi la gestion du pâturage et des ressources fourragères (des brebis taries peuvent supporter un chargement élevé ou valoriser des pâturages médiocres), ainsi que la gestion du troupeau (état corporel des brebis à la lutte, possibilité d'accélération du rythme d'agnelage sur des brebis taries précocement).

\section{Matériel et méthodes}

\section{A. Conduite de l'expérimentation}

L'expérimentation a été répétée au cours de deux années, 1981 et 1982 . Nous avons utilisé 193 agneaux de race Ile-de-France ; 80 mâles en 1981,50 mâles et 63 femelles en 1982.

La prolificité du troupeau expérimental a été de 2,15 en 1981 et de 2,16 en 1982 ; le nombre moyen d'agneaux allaités par brebis s'est élevé à 1,26 en 1981 et 1,43 en 1982.

Les agneaux en expérience sont nés en moyenne le 14 mars en 1981 (du 4 mars au 3 avril), et le 25 février en 1982 (du 23 février au 2 mars).

La mise à l'herbe a eu lieu le 20 avril en 1981 et le 13 avril en 1982.

Le protocole expérimental est résumé sur le tableau 2. Il comprend quatre traitements : sevrage à $6,12,16$ semaines et traitement témoin non sevré. Compte tenu du système de conduite, les animaux sevrés à 6 semaines ne sont pas sortis à l'herbe, ceux qui ont été sevrés plus tard ont pâturé depuis l'âge de 6 semaines jusqu'au sevrage.

En 1981, le choix des agneaux à sevrer s'est fait en retirant de la mère des agneaux représentatifs de ceux présents au moment du tri, sur la base de leur poids le jour du tri. Après sevrage, les agneaux ont été répartis en trois lots d'engraissement, selon leur vitesse de croissance entre la naissance et le sevrage (lente, moyenne, rapide).

La finition de certains agneaux sous la mère avant l'âge de 16 semaines et le protocole de choix des agneaux à sevrer à 16 semaines (représentatifs de ceux présents au pâturage au moment du tri), nous ont conduit à constituer un lot d'agneaux non sevrés comparables à ceux des agneaux à croissance rapide sevrés à 6,12 et 16 semaines. Le lot d'agneaux non sevrés a donc été considéré comme un lot d'agneaux à vitesse de croissance rapide.

En 1982, nous avons constitué, à la mise à l'herbe, deux populations d'agneaux selon que leur vitesse de croissance entre la naissance et la mise à l'herbe était inférieure ou supérieure à $200 \mathrm{~g} /$ jour. Les populations d'agneaux à croissance lente et rapide constituaient respectivement 43 p. 100 et 57 p. 100 du troupeau expérimental. Le tri pour le sevrage s'est fait, à l'intérieur de chaque population et pour chaque sexe, sur la base du poids à la naissance et du poids au moment du tri, de manière à retirer de la mère des agneaux représentatifs de la population. Contrairement à l'année précédente, le choix des agneaux à sevrer à 16 semaines s'est fait en prenant en considération les agneaux finis sous la mère avant l'âge de 16 semaines. 
Les agneaux ont été finis en bergerie après sevrage. De manière à tenir compte des différences de poids de contenu digestif entre les animaux finis à l'herbe et ceux finis en bergerie, les animaux ont été abattus dès que leur poids atteignait :

- $42 \mathrm{~kg}$ et $40 \mathrm{~kg}$ pour les mâles et les femelles finis à l'herbe ;

- $40 \mathrm{~kg}$ et $38 \mathrm{~kg}$ pour les mâles et les femelles finis en bergerie.

Les animaux de renouvellement étaient sortis d'expérience lorsqu'ils atteignaient ce poids.

Les résultats de la première année d'expérience nous ont permis de modifier légèrement le protocole l'année suivante : le sevrage à 6 semaines n'a pas été effectué sur les animaux à croissance rapide. En outre, le sevrage à 16 semaines n'a pas été réalisé sur les agneaux à croissance lente en 1982, à cause de l'effectif réduit en animaux.

L'origine et les caractéristiques des 22 lots expérimentaux constitués au cours des deux années sont résumées sur le tableau 2.

\section{TABLEaU 2}

Lots expérimentaux : caractéristiques et effectifs.

Experimental groups of animals : characteristics and number of animals.

\begin{tabular}{|c|c|c|c|c|c|c|c|}
\hline \multirow[b]{2}{*}{$\begin{array}{l}\text { Année } \\
\text { Year }\end{array}$} & \multicolumn{3}{|c|}{$\begin{array}{l}\text { Age au sevrage (semaines) } \\
\text { Age at weaning (weeks) }\end{array}$} & \multirow[b]{2}{*}{6} & \multirow[b]{2}{*}{12} & \multirow[b]{2}{*}{16} & \multirow[b]{2}{*}{$\begin{array}{c}\text { Pas de } \\
\text { sevrage } \\
\text { Not weaned }\end{array}$} \\
\hline & $\begin{array}{l}\text { Sexe } \\
\text { Sex } \\
\text { (1) }\end{array}$ & $\begin{array}{r}\text { Croissa } \\
\text { av } \\
\text { Lam } \\
\text { bef }\end{array}$ & $\begin{array}{l}\text { 'agneau } \\
\text { ge } \\
\text { rate } \\
\text { ing }\end{array}$ & & & & \\
\hline \multirow[t]{3}{*}{1981} & $\mathbf{M}$ & Lente & Low & 8 & 5 & 7 & \\
\hline & $\mathbf{M}$ & Moyenne & Middle & 8 & 5 & 7 & \\
\hline & $\mathbf{M}$ & Rapide & High & 8 & 5 & 5 & 22 \\
\hline \multirow[t]{4}{*}{1982} & M & Lente & Low & 9 & 8 & & 8 \\
\hline & $\mathbf{M}$ & Rapide & High & & 7 & 9 & 9 \\
\hline & $\mathrm{F}$ & Lente & Low & 8 & 8 & & 8 \\
\hline & $\mathrm{F}$ & Rapide & High & & 13 & 13 & 13 \\
\hline
\end{tabular}

(1) M : Mâles - Males.

F : Femelles - Females.

(2) 1981 : Les lots à croissance lente, moyenne et rapide sont constitués chacun par le tiers des agneaux du lot sevré à l'âge indiqué ; les agneaux sont répartis sur la base de leur cro:ssance ent e la naissance et le sevrage.

Groups characterized by low, middle and high growth rate before weaning are constituted by the third of the group being weaned; animals are selected on the basis of growih rate between birth and weaning.

1982 : Les lots à croissance lente et rapide sont constitués par les animaux dont la croissance entre la naissance et la mise à liherbe est respectivement inférieure et supérieure à $200 \mathrm{~g} / \mathrm{j}$. Groups characterized by low and high growth rate before weaning are constituted by animals whose growth rate between birth and turning out to grass is respectively lower or higher than $200 \mathrm{~g} / \mathrm{d}$. 


\section{B. Alimentation}

Durant les six premières semaines de lactation en bergerie, les brebis ont reçu une ration à base de foin en 1981 (1,5 kg/brebis/jour), à base d'ensilage d'herbe et de foin à volonté en 1982. Cette ration était complétée par une distribution d'aliment concentré en 1981 ( $500 \mathrm{~g} /$ brebis/jour), de maïs grain en 1982 (400 g MS/brebis/jour). Au pâturage, les brebis n'ont reçu aucune complémentation.

Les animaux ont pâturé en rotation des prairies naturelles; la disponibilité en herbe n'a jamais été limitante. Le temps de présence par parcelle a varié de 2 à 12 jours ( 7 jours en moyenne).

Dès l'âge de 15 jours et jusqu'au sevrage, les agneaux ont eu libre accès à un distributeur sélectif contenant du maïs grain entier, conservé humide à l'acide propionique.

Après sevrage, ils ont disposé, à volonté, de rations composées pour 20 p. 100 de foin de pré de deuxième coupe de très bonne qualité et pour 80 p. 100 d'un aliment concentré (composé de 79,5 p. 100 de maïs, 14 p. 100 de tourteau de soja, 1 p. 100 de chlorure d'ammonium, 1 p. 100 d'urée, 1 p. 100 de sel marin, 1 p. 100 de mélasse, 2,5 p. 100 de condiment minéral et vitaminique et dosant 1,03 UFV et 17,1 g de MAT par $\mathrm{kg}$ de produit brut). Les rations étaient préparées deux fois par semaine; une partie était distribuée chaque jour. Lors de la préparation des rations, un échantillon de chaque aliment était prélevé pour déterminer la matière sèche des offerts et des refus.

\section{Etat sanitaire et traitements}

Les agneaux présents au pâturage ont été traités contre le ténia à intervalles réguliers de 4 semaines. Un traitement contre les strongles a été effectué sur ces animaux début juin.

Lors du sevrage, les agneaux ont été soumis à un déparasitage complet (ténia, strongles et coccidies).

Une erreur de fabrication de l'aliment d'engraissement utilisé en 1982 a entraîné une intoxication par le cuivre (teneur de l'aliment : $23 \mathrm{ppm}$ de $\mathrm{Cu}$ ) et la perte de 5 agneaux dans les lots sevrés à 6 semaines.

\section{Mesures}

\section{Evolution de poids}

Les agneaux ont été pesés régulièrement à heure fixe, à intervalles de 14 jours au pâturage et de 7 jours en bergerie.

En 1982, les brebis ont été pesées une semaine après la mise à l'herbe, le jour des différents sevrages, et pour les mères des agneaux non sevrés, le jour de l'abattage de ceux-ci. 


\section{Quantités d'aliments secs ingérées}

Seules ont été mesurées les consommations d'aliments conservés (complément au pâturage, concentré et foin en bergerie).

Les quantités ingérées par animal ont été obtenues de la manière suivante :

\section{a) Maïs grain consommé au pâturage}

Les quantités ingérées au pâturage ont été estimées à partir de mesures hebdomadaires des quantités offertes et refusées et de la teneur en matière sèche des offerts et des refus.

Les différents lots n'étant pas séparés au pâturage, les consommations hebdomadaires de chaque animal ont été calculées sur la base d'une hypothèse d'ingestion identique par $\mathrm{kg}$ de poids vif, quels que soient le sexe et le poids de l'animal. Une expérience ultérieure (PRACHE, non publié) conduite en séparant des agneaux allaités simples et allaités doubles au pâturage, a permis de confirmer la validité de cette hypothèse.

\section{b) Aliment concentré et foin}

Les quantités de concentré et de foin ingérées par les agneaux après sevrage ont été calculées à partir de la mesure bihebdomadaire des quantités offertes et refusées, et de la teneur en matière sèche des offerts et des refus. Les agneaux étant regroupés en lots expérimentaux, la quantité de concentré et de foin consommée par animal a été considérée comme égale à la quantité totale consommée par le lot, divisée par le nombre d'agneaux du lot. Cette quantité a été augmentée, pour chaque individu, de sa consommation au cours de la semaine de sevrage, période pendant laquelle les animaux n'étaient pas encore répartis dans les différents lots d'engraissement. Cette dernière a été calculée sur la base d'une hypothèse d'ingestion par $\mathrm{kg}$ de poids vif, durant la semaine de sevrage, égale à 70 p. 100 de l'ingestion par $\mathrm{kg}$ de poids vif durant la semaine qui a suivi le sevrage.

Les quantités de concentré et de foin ingérées entre la naissance et la mise à l'herbe n'ont pas été mesurées.

\section{Disponibilités en herbe}

La quantité d'herbe offerte a été estimée en 1982, à chaque entrée des animaux sur une parcelle, à partir de la fauche à la minitondeuse de 5 échantillons d'herbe répartis au hasard sur la parcelle. La forme des échantillons était une bande de $10,4 \mathrm{~cm}$ de large sur $2 \mathrm{~m}$ de long; la hauteur de la barre de coupe était de $1,5 \mathrm{~cm}$.

\section{Qualité de la carcasse}

Les mesures suivantes ont été faites :

- le jour de l'abattage : pesée de l'animal vif, du tube digestif plein et vide, et du gras péristomacal;

- après $24 \mathrm{~h}$ de conservation à $+4^{\circ} \mathrm{C}$ : pesée de la carcasse et du gras périrénal, mesure de l'épaisseur du gras dorsal, et, en 1982, appréciation de la qualité du gras dorsal (note variant de 3 pour un gras huileux à 15 pour un gras dur). 


\section{Résultats}

Les conditions climatiques ont été très différentes entre les deux années ; 1982 a été plus chaude et beaucoup plus sèche que 1981 (la température moyenne entre avril et septembre a été de $12,2^{\circ} \mathrm{C}$ contre $11,1^{\circ} \mathrm{C}$; les précipitations durant cette même période se sont élevées à $305 \mathrm{~mm}$ contre $628 \mathrm{~mm}$ ).

\section{A. Quantité d'herbe offerte aux animaux}

En 1982, la quantité d'herbe offerte par brebis suitée et par jour a été de $6,9 \mathrm{~kg}$ MS, en moyenne sur toute la période de pâturage.

\section{B. Variation de poids des brebis}

Les variations de poids des brebis durant la phase de pâturage sont reportées au tableau 3. Elles ont été faibles; les brebis taries le plus tardivement (mères des agneaux à croissance lente non sevrés) ont perdu, en moyenne, $450 \mathrm{~g}$ entre la mise à l'herbe et le tarissement.

\section{TABLEAU 3}

Variation du poids des brebis durant la phase de pâturage.

Changes in ewe body weight during the grazing period.

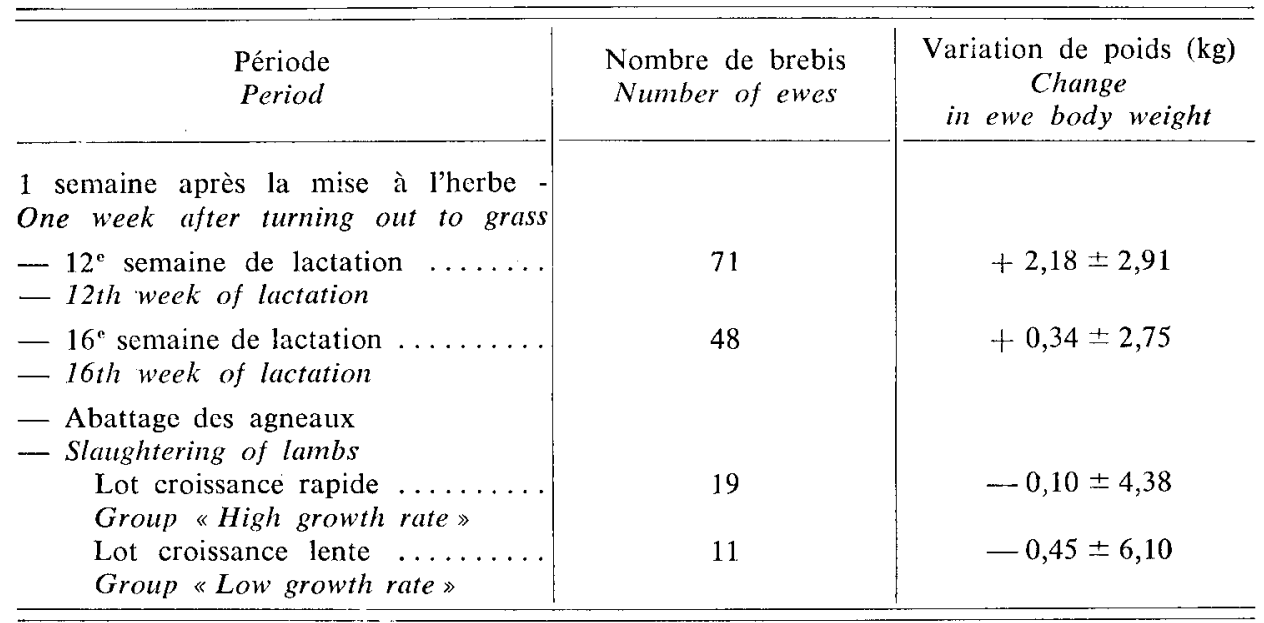

\section{Croissance et âge à l'abattage des agneaux}

(Voir tableaux 4 et 5)

La vitesse de croissance moyenne de l'ensemble des agneaux a atteint $247 \mathrm{~g} / \mathrm{j}$ au cours de la phase d'élevage sous la mère et $331 \mathrm{~g} / \mathrm{j}$ au cours de la phase de finition 


\begin{tabular}{|c|c|c|c|c|c|c|c|c|}
\hline \multirow{4}{*}{ 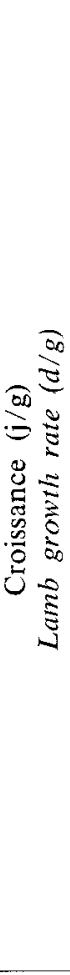 } & 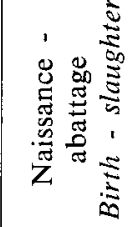 & \multicolumn{3}{|c|}{ 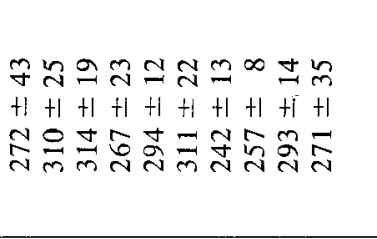 } & \multicolumn{3}{|c|}{ 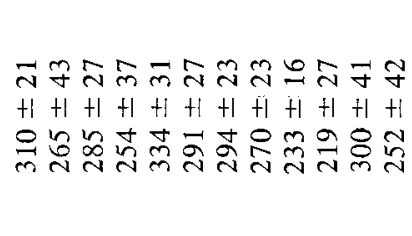 } & \\
\hline & 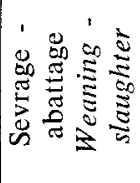 & \multicolumn{3}{|c|}{ 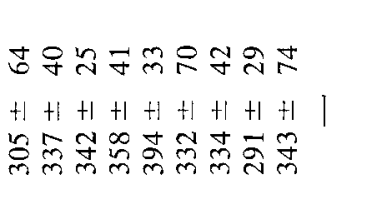 } & \multicolumn{3}{|c|}{ 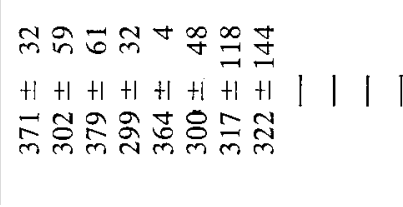 } & \\
\hline & 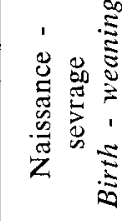 & \multicolumn{3}{|c|}{ 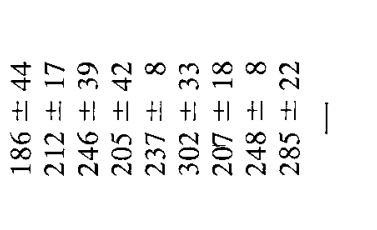 } & \multicolumn{3}{|c|}{ 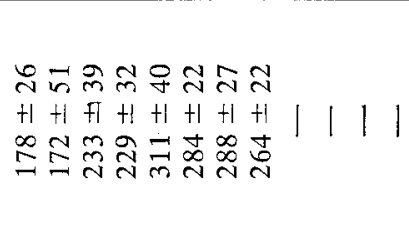 } & \\
\hline & 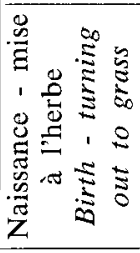 & \multicolumn{3}{|c|}{ 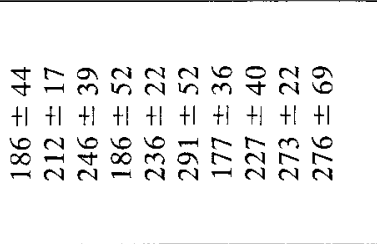 } & \multicolumn{3}{|c|}{ 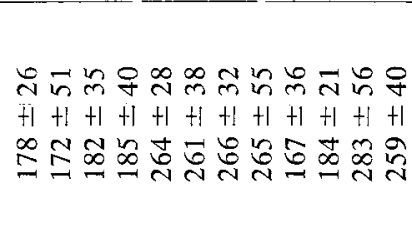 } & $\ddot{0}$ \\
\hline \multicolumn{2}{|c|}{ 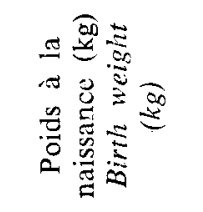 } & \multicolumn{3}{|c|}{ 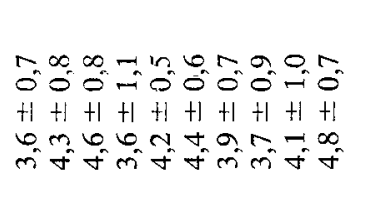 } & \multicolumn{3}{|c|}{ 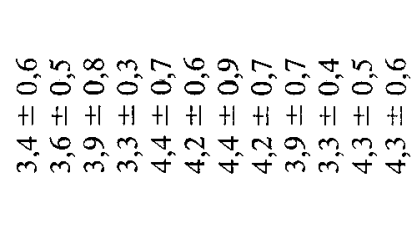 } & 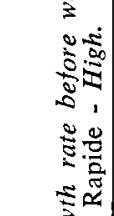 \\
\hline \multicolumn{2}{|c|}{ 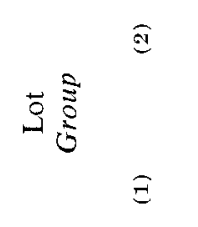 } & \multicolumn{3}{|c|}{$\leadsto \Sigma \simeq \leadsto \Sigma \simeq \mapsto \Sigma \simeq \simeq$} & \multicolumn{3}{|c|}{ 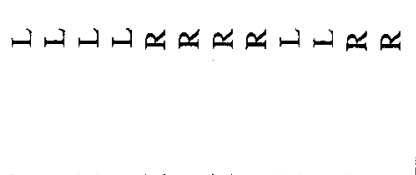 } & 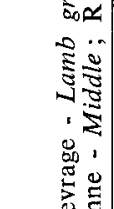 \\
\hline \multicolumn{2}{|c|}{ 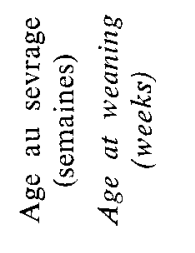 } & \multicolumn{3}{|c|}{ 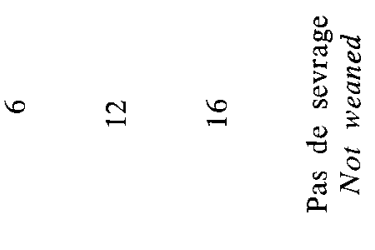 } & \multicolumn{3}{|c|}{ 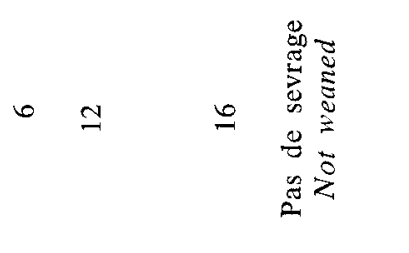 } & 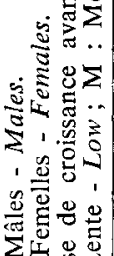 \\
\hline \multicolumn{2}{|r|}{ 导 さ } & \multicolumn{3}{|c|}{$\stackrel{\infty}{\varrho}$} & \multicolumn{3}{|c|}{$\stackrel{1}{\infty}$} & 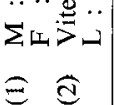 \\
\hline
\end{tabular}




\section{Tableau 5}

Age à l'abattage des animaux

(ou de sortie d'expérience pour les animaux de renouvellement).

Age at slaughter.

\begin{tabular}{|c|c|c|c|c|}
\hline $\begin{array}{l}\text { Année } \\
\text { Year }\end{array}$ & $\begin{array}{l}\text { Age au sevrage } \\
\text { (semaines) } \\
\text { Age at weaning } \\
\text { (weeks) }\end{array}$ & \multicolumn{2}{|c|}{$\begin{array}{l}\text { Lot } \\
\text { Group }\end{array}$} & $\begin{array}{l}\text { Age à l'abattage (jours) } \\
\text { Age at slaughter (days) }\end{array}$ \\
\hline \multirow[t]{4}{*}{1981} & 6 & $\begin{array}{l}\mathbf{M} \\
\mathbf{M} \\
\mathbf{M}\end{array}$ & $\begin{array}{l}\mathrm{L} \\
\mathrm{M} \\
\mathrm{R}\end{array}$ & $\begin{array}{l}142 \pm 19 \\
128 \pm 10 \\
123 \pm 6\end{array}$ \\
\hline & 12 & $\begin{array}{l}\mathbf{M} \\
\mathbf{M} \\
\mathbf{M}\end{array}$ & $\begin{array}{r}\mathrm{L} \\
\mathrm{M} \\
\mathrm{R}\end{array}$ & $\begin{array}{l}138 \pm 17 \\
129 \pm 9 \\
120 \pm 4\end{array}$ \\
\hline & 16 & $\begin{array}{l}\mathbf{M} \\
\mathbf{M} \\
\mathbf{M}\end{array}$ & $\begin{array}{r}\mathbf{L} \\
\mathbf{M} \\
\mathbf{R}\end{array}$ & $\begin{array}{l}155 \pm 4 \\
149 \pm 5 \\
136 \pm 6\end{array}$ \\
\hline & $\begin{array}{l}\text { Pas de sevrage } \\
\text { Not weaned }\end{array}$ & $\mathbf{M}$ & $\mathbf{R}$ & $142 \pm 19$ \\
\hline \multirow[t]{4}{*}{1982} & 6 & $\begin{array}{l}\mathrm{M} \\
\mathrm{F}\end{array}$ & $\begin{array}{l}\mathrm{L} \\
\mathbf{L}\end{array}$ & $\begin{array}{l}120 \pm 8 \\
122 \pm 10\end{array}$ \\
\hline & 12 & $\begin{array}{l}\text { M } \\
\text { F } \\
\text { M } \\
\text { F }\end{array}$ & $\begin{array}{l}\text { L } \\
\text { L } \\
\text { R } \\
\text { R }\end{array}$ & $\begin{array}{l}126 \pm 12 \\
133 \pm 16 \\
108 \pm 9 \\
114 \pm 11\end{array}$ \\
\hline & 16 & $\begin{array}{l}\text { M } \\
\text { F }\end{array}$ & $\begin{array}{l}\mathrm{R} \\
\mathrm{R}\end{array}$ & $\begin{array}{l}126 \pm 8 \\
128 \pm 8\end{array}$ \\
\hline & $\begin{array}{l}\text { Pas de sevrage } \\
\text { Not weaned }\end{array}$ & $\begin{array}{l}\text { M } \\
F \\
\text { M } \\
\text { F }\end{array}$ & $\begin{array}{l}\mathrm{L} \\
\mathrm{L} \\
\mathrm{R} \\
\mathrm{R}\end{array}$ & $\begin{array}{l}166 \pm 13 \\
160 \pm 20 \\
129 \pm 19 \\
139 \pm 20\end{array}$ \\
\hline
\end{tabular}

(1) M : Mâles - Males.

F : Femelles - Females.

(2) Vitesse de croissance avant sevrage - Lamb growth rate before weaning :

L : Lente - Low; M : Moyenne - Middle; R : Rapide - High.

en bergerie. Compte tenu d'une vitesse de croissance moyenne de $277 \mathrm{~g} / \mathrm{j}$ entre la naissance et l'abattage, l'âge moyen à l'abattage a été de 133 jours. Pour un même régime expérimental, les croissances ont été voisines entre les deux années. 


\section{Croissance}

\section{a) Croissance au cours de la phase lactée}

La croissance au cours de la phase lactée varie très significativement avec le poids à la naissance et le nombre d'agneaux allaités.

Une analyse de variance-covariance montre :

- qu'à même mode d'allaitement, un écart de poids de $1 \mathrm{~kg}$ à la naissance se traduit par un écart de poids de $1,92 \mathrm{~kg}, 2,26 \mathrm{~kg}$ et $1,90 \mathrm{~kg}$ à 6,12 et 16 semaines respectivement ;

- qu'à même poids à la naissance, un agneau allaité simple pèse $2,44 \mathrm{~kg}$, $3,70 \mathrm{~kg}$ et $2,91 \mathrm{~kg}$ de plus à 6,12 et 16 semaines respectivement qu'un agneau allaité double.

D'autre part, la vitesse de croissance au cours de la phase de pâturage varie très significativement avec la vitesse de croissance au cours des six premières semaines de vie en bergerie. Les équations du tableau 6 montrent qu'une différence de $100 \mathrm{~g} / \mathrm{j}$ pendant les six premières semaines de vie, s'est traduite, pour les agneaux sous la mère, par une différence de $37 \mathrm{~g} / \mathrm{j}$ entre la mise à l'herbe et l'âge de 12 semaines, de $19 \mathrm{~g} / \mathrm{j}$ entre la mise à l'herbe et l'âge de 16 semaines, et pour les agneaux non sevrés, de $29 \mathrm{~g} / \mathrm{j}$ entre la mise à l'herbe et l'abattage.

\section{b) Croissance après sevrage}

Quels que soient le sexe, la vitesse de croissance sous la mère et l'âge au sevrage, la vitesse de croissance des agneaux augmente après sevrage, lorsqu'ils passent d'un régime essentiellement lacté (sevrage à 6 semaines) ou mixte (lait, herbe et concentré pour les sevrages plus tardifs) au régime d'engraissement. Cette augmentation de la vitesse de croissance qui est en moyenne de $84 \mathrm{~g} / \mathrm{j}$ pour l'ensemble des agneaux (247 g/j en moyenne avant sevrage et $331 \mathrm{~g} / \mathrm{j}$ en moyenne après sevrage), est d'autant plus importante que le sevrage est plus précoce, et que la croissance de l'agneau sous la mère est faible (de 70 à $193 \mathrm{~g} / \mathrm{j}$ et de 16 à $96 \mathrm{~g} / \mathrm{j}$ respectivement pour les lots d'agneaux à vitesse de croissance lente et rapide avant sevrage).

D'autre part, l'étude de la relation entre la vitesse de croissance de la naissance au sevrage - GMQ 1 - et la vitesse de croissance du sevrage à l'abattage (ou la sortie d'expérience) - GMQ 2 - montre que, pour chaque traitement (sevrage à 6, 12 ou 16 semaines), ces deux variables sont indépendantes. Ceci signifie, que, pour chaque traitement, la vitesse de croissance après le sevrage ne dépend pas de la vitesse de croissance avant celui-ci ; la courbe de régression de GMQ 2 en GMQ 1 , ajustée pour l'année, est une droite horizontale d'ordonnée $333 \mathrm{~g} / \mathrm{j}$ pour le traitement 6 semaines $(n=41), 357 \mathrm{~g} / \mathrm{j}$ pour le traitement 12 semaines $(\mathrm{n}=51)$ et $320 \mathrm{~g} / \mathrm{j}$ pour le traitement 16 semaines $(n=41)$.

Ce résultat est illustré à la figure 1 qui concerne les agneaux sevrés à 6 semaines en 1981.

\section{Age à l'abattage}

Quels que soient l'année et le sexe, et pour chaque type de croissance (lente, moyenne, ou rapide) : 


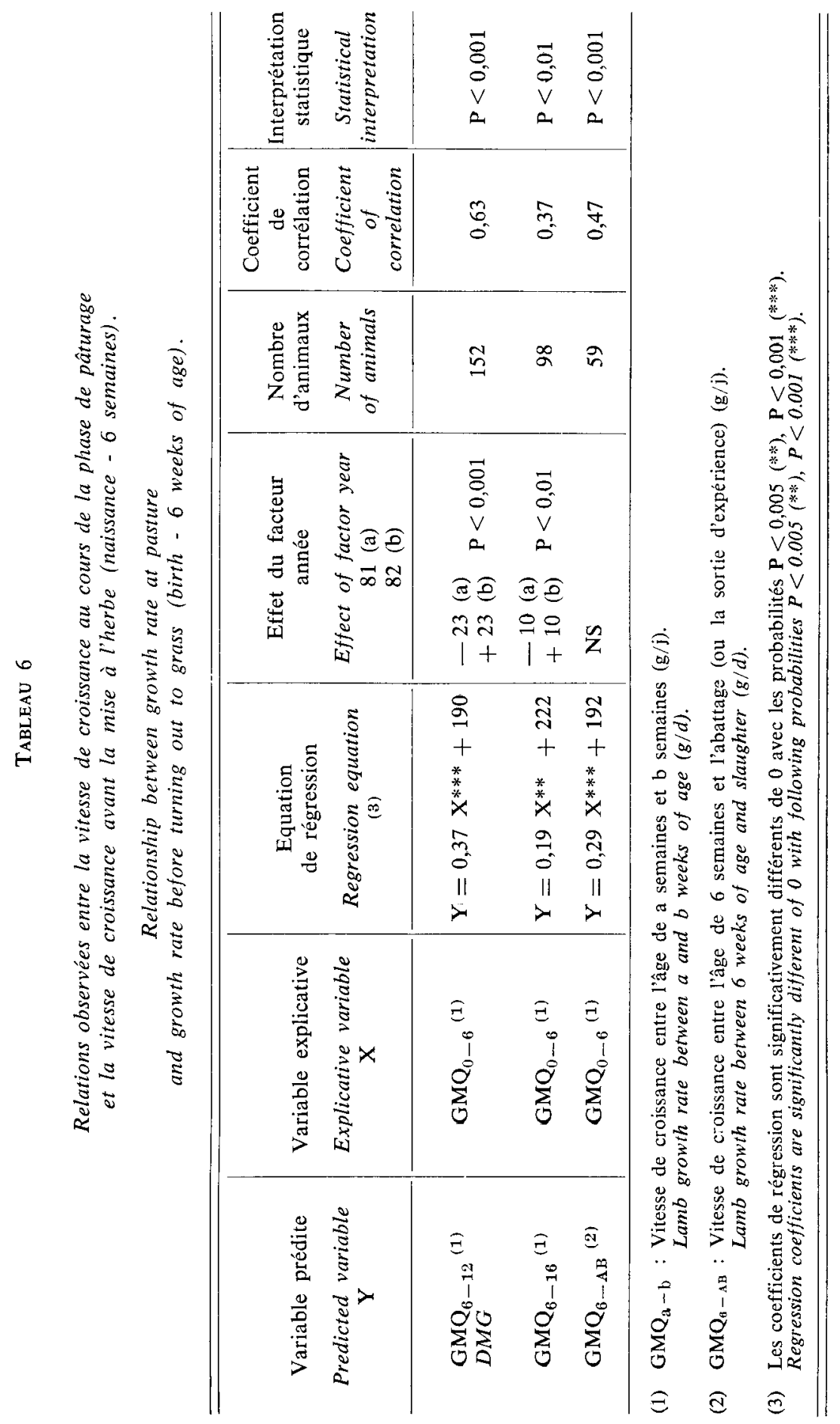


- les animaux sevrés ont toujours été abattus plus jeunes que les animaux non sevrés. La différence est très significative pour les lots sevrés à 6 semaines $(P<0,001)$ et à 12 semaines $(\mathrm{P}<0,001$ à $\mathrm{P}<0,005)$; elle l'est moins pour les lots sevrés à 16 semaines $(P<0,05$ à $N S)$;

- l'âge à l'abattage n'est jamais significativement différent entre les lots sevrés à 6 semaines et les lots sevrés à 12 semaines;

- les animaux sevrés à 16 semaines ont toujours été abattus plus âgés que les animaux sevrés à 6 semaines ( $\mathrm{P}<0,005$ à $\mathrm{NS}$ ) et 12 semaines $(\mathrm{P}<0,025$ à $\mathrm{NS}$ ).

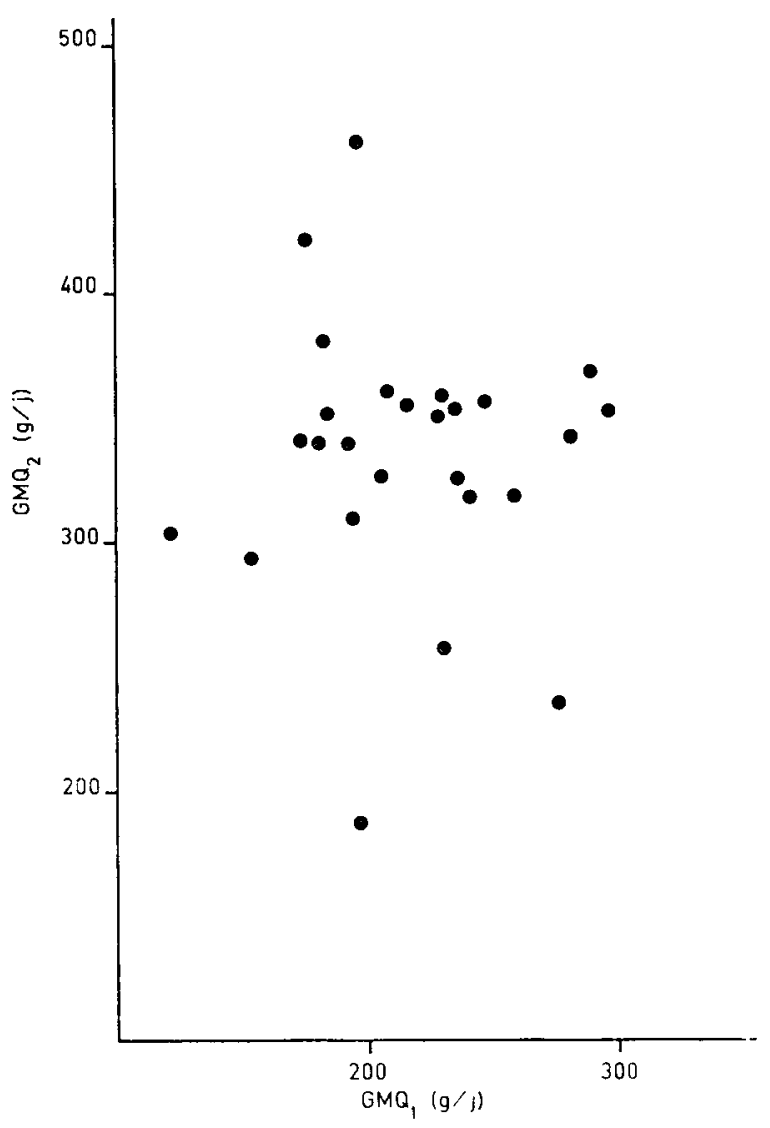

Fig. 1

Relation entre la vitesse de croissance avant sevrage - GMQ et la vitesse de croissance après sevrage $-G M Q_{2}-$ (animaux sevrés à 6 semaines en 1981).

Relationship between growth rate before weaning - $D M G_{1}-$ and growth rate after weaning - DMG. - (animals weaned at 6 weeks in 1981).

\section{Consommations d'aliments conservés}

Les quantités d'aliment ingérées par animal (complément au pâturage, concentré et foin en bergerie) sont reportées au tableau 7 . 


\section{TABLEAU 7}

Quantités ingérées.

Feed intake.

\begin{tabular}{|c|c|c|c|c|c|c|}
\hline \multirow{3}{*}{$\begin{array}{l}\text { Année } \\
\text { Year }\end{array}$} & \multirow{3}{*}{$\begin{array}{l}\text { Age au sevrage } \\
\text { (semaines) } \\
\text { Age at weaning } \\
\text { (weeks) }\end{array}$} & & & \multicolumn{3}{|c|}{$\begin{array}{l}\text { Quantités ingérées (UFV/agneau) } \\
\text { Feed intake (UFV per lamb) }\end{array}$} \\
\hline & & \multicolumn{2}{|c|}{$\begin{array}{c}\text { Lot } \\
\text { Group }\end{array}$} & \multicolumn{2}{|c|}{$\begin{array}{l}\text { Concentré } \\
\text { Concentrate }\end{array}$} & \multirow{2}{*}{$\begin{array}{l}\text { Foin } \\
\text { Hay }\end{array}$} \\
\hline & & & & $\begin{array}{l}\text { Avant sevrage } \\
\text { Before weaning }\end{array}$ & $\begin{array}{l}\text { Après sevrage } \\
\text { After weaning }\end{array}$ & \\
\hline \multirow[t]{10}{*}{1981} & 6 & $\mathbf{M}$ & $\mathrm{L}$ & & 95,8 & 17,8 \\
\hline & & M & $\overline{\mathbf{M}}$ & & 94,0 & 16,2 \\
\hline & & $\mathbf{M}$ & $\mathbf{R}$ & & 79,4 & 14,4 \\
\hline & 12 & M & $\mathrm{L}$ & 5,1 & 56,1 & 9,7 \\
\hline & & M & $\mathbf{M}$ & 6,0 & 57,2 & 9,0 \\
\hline & & M & $\mathbf{R}$ & 7,5 & 39,2 & 6,7 \\
\hline & 16 & $\mathbf{M}$ & $\mathrm{L}$ & 13,0 & 55,7 & 9,6 \\
\hline & & M & $\mathbf{M}$ & 15,2 & 36,9 & 7,1 \\
\hline & & $\mathbf{M}$ & $\mathbf{R}$ & 17,5 & & 2,7 \\
\hline & $\begin{array}{l}\text { Pas de sevrage } \\
\text { Not weaned }\end{array}$ & M & $\mathbf{R}$ & 35,4 & & \\
\hline \multirow[t]{12}{*}{1982} & 6 & M & $\mathrm{L}$ & & 80,5 & 12,7 \\
\hline & & $\mathrm{F}$ & $\mathbf{L}$ & & 89,2 & 13,0 \\
\hline & 12 & $\mathbf{M}$ & $\mathrm{L}$ & 3,3 & 59,3 & 8,3 \\
\hline & & $\mathrm{F}$ & $\mathrm{L}$ & 3,3 & 62,0 & 9,9 \\
\hline & & M & $\mathrm{R}$ & 4,5 & 33,3 & 4,3 \\
\hline & & $\mathbf{F}$ & $\mathrm{R}$ & 4,2 & 39,7 & 5,9 \\
\hline & 16 & M & $\mathbf{R}$ & 12,2 & 15,8 & 2,7 \\
\hline & & F & $\mathbf{R}$ & 11,6 & 18,9 & 3,6 \\
\hline & Pas de sevrage & $\mathbf{M}$ & $\mathrm{L}$ & 45,3 & & \\
\hline & Not weaned & $\mathrm{F}$ & $\mathrm{L}$ & 38,7 & & \\
\hline & & $\mathbf{M}$ & $\vec{R}$ & 31,0 & & \\
\hline & & $\mathrm{F}$ & $\mathbf{R}$ & 30,8 & & \\
\hline
\end{tabular}

(1) M : Mâles - Males.

$\mathrm{F}$ : Femelles - Females.

(2) Vitesse de croissance avant sevrage - Lamb growth rate before weaning :

$\mathrm{L}$ : Lente - Low ; M : Moyenne - Middle; R : Rapide - High.

Pour un traitement comparable, elles ont été voisines entre les deux années, bien qu'un peu plus faibles en 1982 ; ceci peut s'expliquer, en partie, par des vitesses de croissance légèrement meilleures en 1982. 
L'effet de l'âge au sevrage et de la croissance de l'agneau sous la mère sur la consommation d'aliments à forte concentration énergétique sont illustrés au tableau 8 . Pour un âge au sevrage donné, les quantités d'aliment concentré ingérées sont d'autant plus importantes que la croissance de l'agneau sous la mère a été faible.

\section{TABLeaU 8}

Effet de l'âge au sevrage sur le niveau d'ingestion d'aliments à forte concentration énergétique (UFV/kg de poids vif à l'abattage ou à la sortie d'expérience).

Effect of the age at weaning on concentrate intake (UFV/kg live weight at slaughter).

\begin{tabular}{|c|c|c|c|c|c|c|}
\hline \multirow{2}{*}{$\begin{array}{l}\text { Année } \\
\text { Year }\end{array}$} & \multirow{2}{*}{$\begin{array}{l}\text { Sexe } \\
\text { Sex }\end{array}$} & \multirow{2}{*}{$\begin{array}{l}\text { Vitesse de croissance } \\
\text { avant sevrage } \\
\text { Lamb growth rate } \\
\text { before weaning }\end{array}$} & \multicolumn{4}{|c|}{$\begin{array}{l}\text { Age au sevrage (semaines) } \\
\text { Age at weaning (weeks) }\end{array}$} \\
\hline & & & 6 & 12 & 16 & $\begin{array}{c}\text { Pas de } \\
\text { sevrage } \\
\text { Not weaned }\end{array}$ \\
\hline 1981 & $\begin{array}{l}\text { Mâles } \\
\text { Males }\end{array}$ & \multirow{3}{*}{$\begin{array}{l}\text { Rapide } \\
\text { High }\end{array}$} & 1,84 & 1,12 & 0,82 & 0,83 \\
\hline 1982 & $\begin{array}{l}\text { Mâles } \\
\text { Males }\end{array}$ & & & 0,94 & 0,68 & 0,72 \\
\hline 1982 & $\begin{array}{l}\text { Femelles } \\
\text { Females }\end{array}$ & & & 1,18 & 0,79 & 0,80 \\
\hline 1981 & $\begin{array}{l}\text { Mâles } \\
\text { Males }\end{array}$ & $\begin{array}{l}\text { Moyenne } \\
\text { Middle }\end{array}$ & 2,20 & 1,50 & 1,24 & \\
\hline 1981 & $\begin{array}{l}\text { Mâles } \\
\text { Males }\end{array}$ & \multirow{3}{*}{$\begin{array}{l}\text { Lente } \\
\text { Low }\end{array}$} & 2,30 & 1,52 & \multirow[t]{3}{*}{1,66} & \\
\hline 1982 & $\begin{array}{l}\text { Mâles } \\
\text { Males }\end{array}$ & & 1,98 & 1,58 & & 1,07 \\
\hline 1982 & $\begin{array}{l}\text { Femelles } \\
\text { Females }\end{array}$ & & 2,39 & 1,73 & & 1,02 \\
\hline
\end{tabular}

Quelle que soit la vitesse de croissance de l'agneau sous la mère, la consommation d'aliment concentré est maximum pour les lots sevrés à 6 semaines ; elle diminue ensuite pour passer par un minimum se situant vers 16 semaines d'âge au sevrage pour les lots d'agneaux à vitesse de croissance rapide sous la mère et 12 semaines pour les lots d'agneaux à vitesse de croissance faible, puis elle augmente légèrement au-delà. Cette évolution apparaît dans tous les lots, à l'exception de ceux à vitesse de croissance initiale lente en 1982. Pour ceux-ci, le calcul de la quantité de concentré ingérée par les agneaux non sevrés entre la mise à l'herbe et l'âge de 16 semaines donne des valeurs comparables à celles des agneaux à vitesse de croissance lente sevrés à 16 semaines en 1981 (7,91 $\mathrm{kg}$ MS en 1982 contre 10,15 kg MS en 1981). L'évolution ultérieure peut être due aux conditions météorologiques particulières de l'année 1982 : des chutes de pluie à partir de la deuxième quinzaine de juillet ont, en effet, entraîné une reprise de la croissance de l'herbe, et les agneaux encore présents au 
pâturage - donc principalement des animaux à croissance initiale lente - ont pu ingérer cette herbe jeune de préférence au maïs ; ceci a entraîné une chute de l'ingestion de maïs, comme on peut l'observer à la figure 2 .

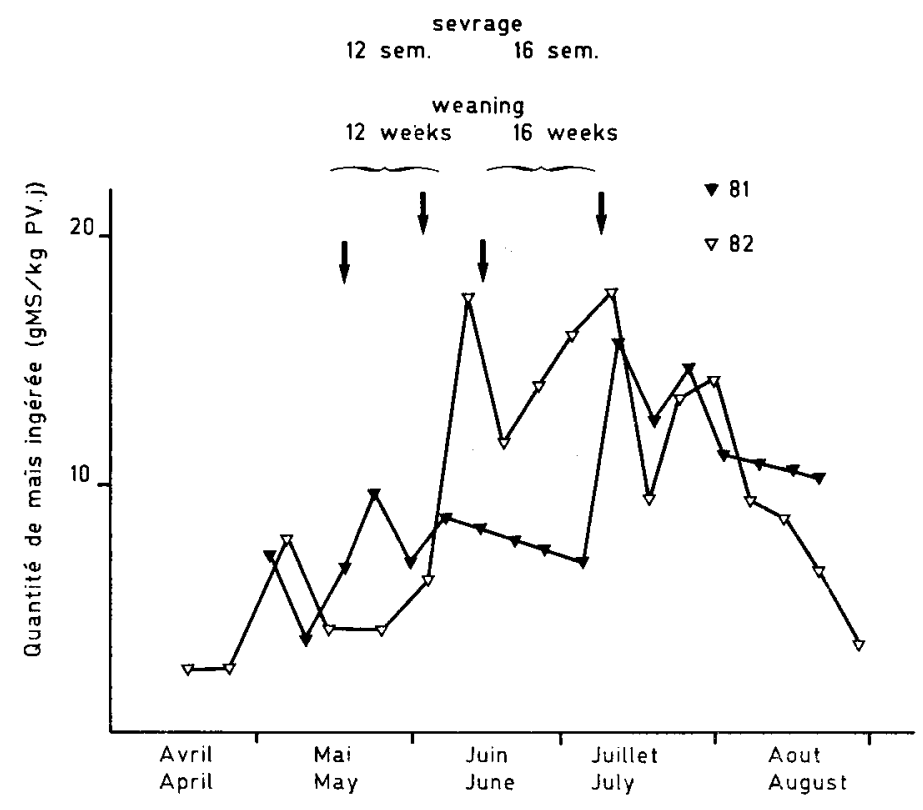

FIG. 2

Quantité de maïs ingérée au cours de la saison de pâturage (g MS/kg PV.j). Maize intake at pasture ( $\mathrm{g} \mathrm{DM} / \mathrm{kg} \mathrm{LW}$. day).

\section{E. Qualité de la carcasse}

Pour l'analyse des données, les animaux ont été regroupés en 14 lots sur la base du protocole de 1982 (constitution de deux populations d'agneaux selon que leur vitesse de croissance initiale - de la naissance à six semaines - est inférieure ou supérieure à $200 \mathrm{~g} / \mathrm{j}$ ).

Les résultats des mesures effectuées à l'abattage (résultats bruts et ajustés par analyse de covariance pour le poids de carcasse froide) sont reportés au tableau 9.

Pour les lots expérimentaux représentés lors des deux années, le poids de carcasse froide et les différents critères d'engraissement ajustés à même poids de carcasse froide n'ont pas été significativement différents entre les deux années.

\section{Effet de l'âge au sevrage}

Quels que soient le sexe, la vitesse de croissance initiale de l'agneau et le crisère d'engraissement, il n'y a généralement pas de différence significative entre les carcasses 


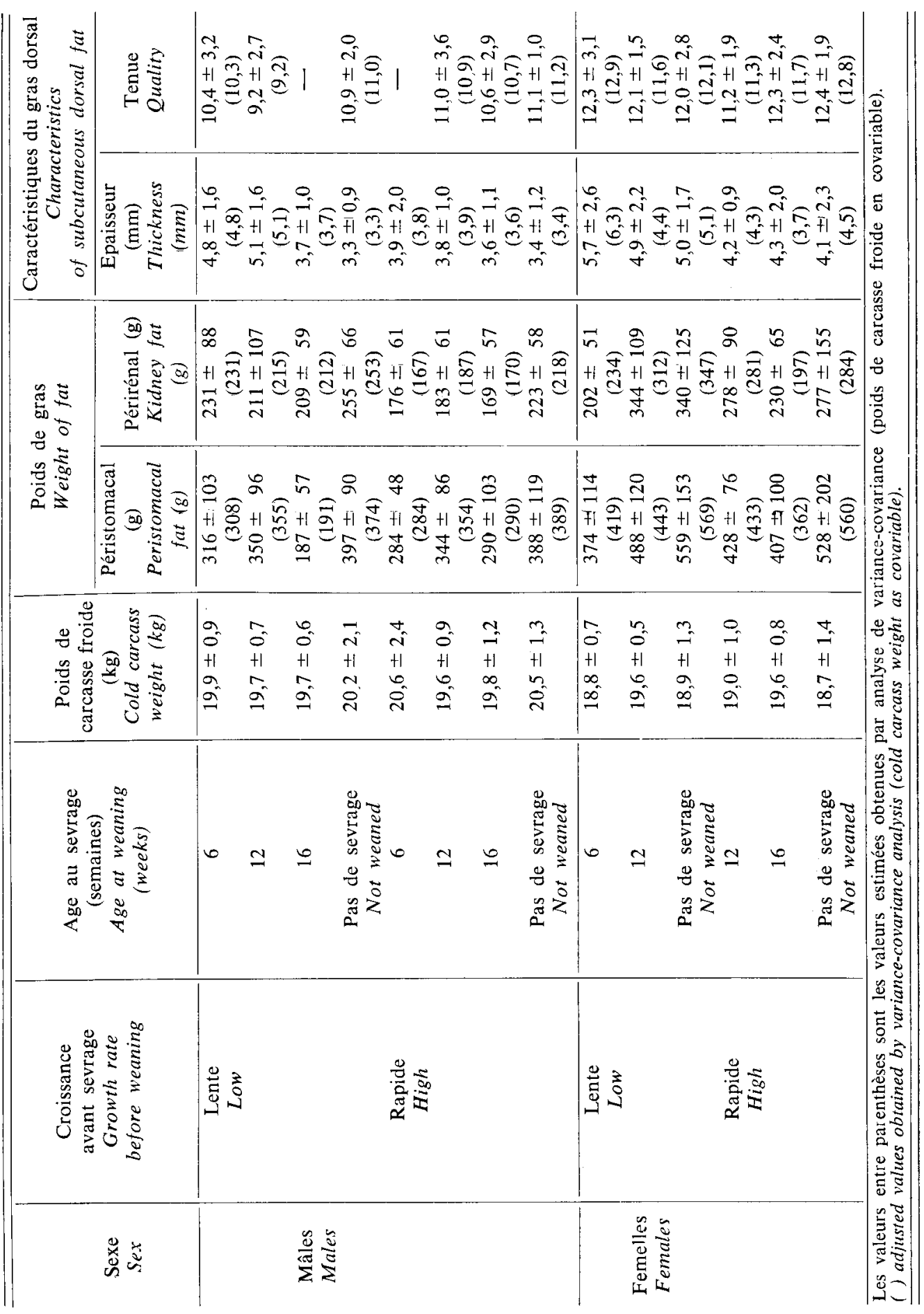




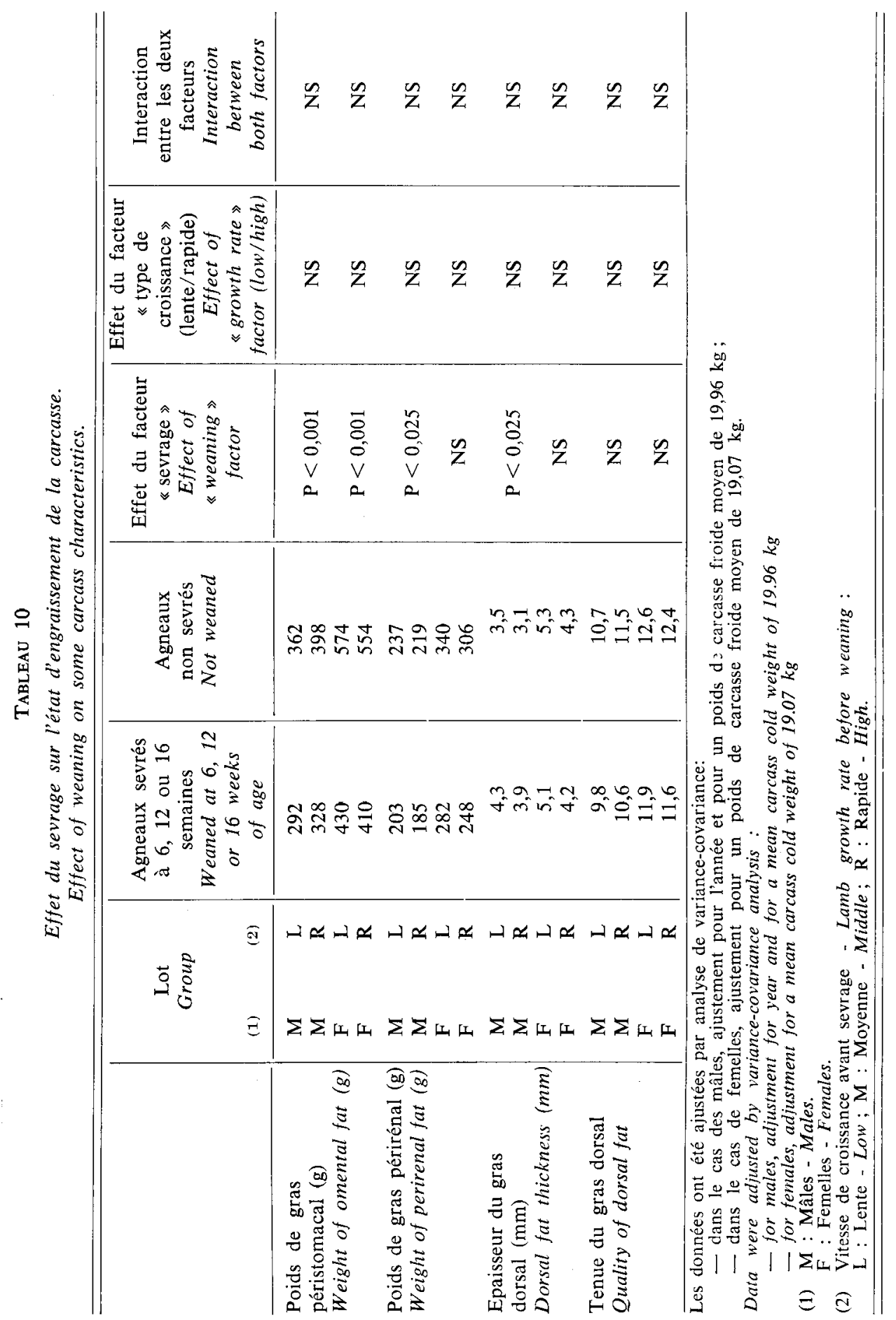


d'agneaux sevrés à 6,12 ou 16 semaines. Toutefois, pour tous les critères d'engraissement, les agneaux sevrés à 16 semaines ont toujours tendance a être moins gras que les agneaux sevrés à 6 et 12 semaines, les différences étant même quelquefois significatives.

\section{Effet du sevrage (voir le tableau 10)}

A même poids de carcasse froide, les agneaux sevrés (à 6,12 ou 16 semaines) ont très significativement moins de gras péristomacal que les agneaux non sevrés (70 $\mathrm{g}-\mathrm{P}<0,001$ et $144 \mathrm{~g}-\mathrm{P}<0,001$ en moins respectivement chez les mâles et les femelles). Ils ont aussi moins de gras périrénal, mais ce résultat est significatif uniquement pour les mâles ( $34 \mathrm{~g}$ en moins $\mathrm{P}<0,025$ ). Les mâles sevrés ont un gras dorsal significativement plus épais que les mâles non sevrés $(0,8 \mathrm{~mm}$ en plus, $\mathrm{P}<0,025)$, mais ceci n'apparaît pas chez les femelles. Il n'y a pas d'effet significatif du sevrage sur la tenue du gras dorsal.

\section{Discussion}

Les quantités de concentré ingérées par les agneaux au cours de ces deux essais sont en général concordantes avec celles citées en introduction. Les observations de FonTANILAS (1971) sur agneaux non sevrés donnent des résultats un peu inférieurs aux nôtres, probablement, en partie, parce que les agneaux ont été conduits en pâturage en avant. D’autres résultats sont plus élevés que les nôtres : ceux de VAN QUACKEBEKE (1976) sur agneaux non sevrés (26 à $59 \mathrm{~kg}$ brut contre 30 à $44 \mathrm{~kg}$ brut dans nos essais), et sur agneaux sevrés vers $110-120$ jours (17 à $31 \mathrm{~kg}$ de concentré brut consommé au pâturage contre 11 à $17 \mathrm{~kg}$ brut dans nos essais). Les données de MAITRE (1980) sur agneaux sevrés à 14 semaines sont beaucoup plus élevées que les nôtres, probablement à cause de faibles vitesses de croissance en bergerie $(232 \mathrm{~g} / \mathrm{j})$, car les consommations qu'il observe au pâturage sont proches des nôtres. Enfin, les données de Maitre (1982), pour différents types de conduite des agneaux élevés à l'herbe, atteignent des niveaux extrêmement élevés $(120 \mathrm{~kg}$ de concentré brut).

L'intérêt de nos résultats est d'expliquer, en partie, la variabilité observée dans ces données bibliographiques : la consommation totale d'aliments concentrés d'un agneau élevé à l'herbe varie avec sa vitesse de croissance sous la mère et avec l'âge auquel il est sevré. Nos résultats montrent que la consommation d'aliment concentré est minimum avec un sevrage réalisé vers 12 semaines pour les agneaux à vitesse de croissance lente sous la mère et vers 16 semaines pour les agneaux à vitesse de croissance rapide.

Bien que les données bibliographiques soient rares à ce sujet, d'autres auteurs aboutissent à des conclusions voisines.

Orskov et al. (1971) ont travaillé sur des agneaux doubles dont l'un était sevré à 6,13 ou à 20 semaines et fini en bergerie, alors que l'autre était laissé sous la mère avec de l'herbe à volonté, mais sans complémentation. Les agneaux sevrés à 6 semaines ont consommé significativement plus d'aliment concentré que les animaux sevrés à 13 et 20 semaines; il n'y a pas eu de différence significative entre les 
lots sevrés à 13 et 20 semaines, mais la consommation de concentré la plus faible a été observée pour le lot sevré à 13 semaines $(73,5 \mathrm{~kg}$ MS, 42,2 kg MS, 42,9 kg MS respectivement pour les lots sevrés à 6,13 et 20 semaines).

MARTin (1982), travaillant dans un système de conduite semblable au nôtre, conseille de sevrer vers 10-11 semaines les agneaux à faible vitesse de croissance et de les finir en bergerie; les consommations qu'il obtient sur ce type d'agneau sont proches des nôtres (de 60 à $90 \mathrm{~kg}$ de concentré brut, contre 60 à $63 \mathrm{~kg}$ et 78 à $93 \mathrm{~kg}$ de concentré brut dans nos essais pour les agneaux à faible vitesse de croissance sevrés à 12 et 6 semaines respectivement).

Ces résultats s'expliquent, car même dans des conditions de pâturage très favorables (disponibilité en herbe non limitante, complémentation des agneaux à volonté), la vitesse de croissance des agneaux au cours de la phase de pâturage sous la mère reste très liée à leur vitesse de croissance au cours des six premières semaines de vie en bergerie. Une différence de $100 \mathrm{~g} / \mathrm{j}$ au cours des six premières semaines s'est traduite par une différence de $37 \mathrm{~g} / \mathrm{j}, 19 \mathrm{~g} / \mathrm{j}$ et $29 \mathrm{~g} / \mathrm{j}$ respectivement entre la mise à l'herbe et l'âge de 12, 16 semaines et l'abattage. Ceci met en évidence l'importance de la quantité de lait ingérée sur les performances de croissance des agneaux élevés à l'herbe sous la mère : les agneaux dont la vitesse de croissance au cours des 42 premiers jours de vie a été la plus élevée sont ceux qui ont disposé de la quantité de lait la plus importante, avant la mise à l'herbe, mais également au cours de la phase de pâturage, et ce sont eux dont la vitesse de croissance a été la plus élevée au pâturage.

Cette importance de la quantité de lait ingérée sur les performances de croissance de l'agneau élevé à l'herbe a été bien mise en évidence dans les travaux de PenNing \& GiBB (1979), cités en introduction : même en conditions d'herbe non limitante, la vitesse de croissance d'agneaux allaités artificiellement à différents niveaux de lait était d'autant plus élevée que la quantité de lait ingérée était importante. Une diminution de l'apport de lait provoquait une augmentation de l'ingestion d'herbe, mais, compte tenu de la différence de valeur énergétique entre l'herbe et le lait, cette dernière n'était pas suffisante pour compenser la réduction de l'apport de lait.

Même dans nos conditions où, non seulement les disponibilités en herbe ne sont pas limitantes, mais où les agneaux sont complémentés à volonté au pâturage, l'influence de la quantité de lait ingérée - exprimée par la vitesse de croissance entre la naissance et 42 jours - sur les performances de croissance à l'herbe, a persisté jusqu'à l'abattage.

Par contre, la vitesse de croissance lors de la finition en bergerie ne dépend pas de la vitesse de croissance avant sevrage et atteint des niveaux très élevés quel que soit le type d'agneau sevré (de $291 \mathrm{~g} / \mathrm{j}$ à $394 \mathrm{~g} / \mathrm{j}$ dans nos expériences).

Ceci signifie que le sevrage suivi d'une finition en bergerie se traduit par une augmentation de la vitesse de croissance qui est d'autant plus importante que l'agneau a eu une faible vitesse de croissance sous la mère (dans nos expériences, l'augmentation moyenne de croissance après sevrage a été de $134 \mathrm{~g} / \mathrm{j}$ et de $65 \mathrm{~g} / \mathrm{j}$ respectivement pour les lots d'agneaux à vitesse de croissance lente et rapide sous la mère).

En conséquence, les agneaux sevrés et finis en bergerie ont été abattus plus jeunes que les animaux non sevrés, et ce, d'autant plus que leur vitesse de croissance sous la mère a été faible et que le sevrage a été précoce. La réduction de l’âge à 
l'abattage pour les agneaux à vitesse de croissance lente sous la mère a été de 38 à 46 jours $(P<0,001)$ et de 27 à 40 jours $(P<0,001)$ respectivement pour les lots sevrés à 6 et 12 semaines comparés aux lots non sevrés; pour les agneaux à vitesse de croissance rapide sous la mère, elle a été de 19 jours $(\mathrm{P}<0,001)$, de 21 à 25 jours $(\mathrm{P}<0,001$ à $\mathrm{P}<0,005)$ et de 3 à 11 jours $(\mathrm{P}<0,05$ à $\mathrm{NS})$ respectivement pour les lots sevrés à 6,12 et 16 semaines comparés aux lots non sevrés.

L'ensemble de ces résultats permettent de conclure, que plus un animal a une croissance faible sous la mère, plus il est souhaitable de le sevrer précocement, et qu'inversement il est inutile de sevrer jeune un animal qui a une bonne croissance sous la mère. Les données bibliographiques sur cet aspect sont peu nombreuses. MaITRE (1980) observe, avec un type de conduite identique au nôtre, que la vitesse de croissance au cours de la finition en bergerie après sevrage ne dépend pas de la vitesse de croissance avant sevrage ; il en conclut qu'il est préférable de sevrer les agneaux à faible vitesse de croissance plus jeunes que les agneaux à forte vitesse de croissance. Nos résultats confirment ceux de RutTer (1970), obtenus avec des agneaux à forte vitesse de croissance sous la mère. Il a travaillé sur des agneaux pesant en moyenne $18 \mathrm{~kg}$ en début d'expérience (l'âge des animaux variant de 44 à 61 jours), et comparé la vitesse de croissance d'agneaux sevrés à cette date, rentrés en bergerie et nourris à volonté (lot $\mathrm{C}$ ) avec celle d'agneaux pâturant sous la mère sans complémentation jusqu'au sevrage (lot A). A la fin des dix semaines d'expérience, le gain de poids vif du lot $\mathrm{C}$ était plus faible que le gain de poids vif du lot $\mathrm{A}$; l'auteur en conclut que ce type d'agneau ne doit pas être sevré précocement.

Pratiquement, il existerait donc une croissance seuil au-delà de laquelle il est inutile de sevrer - à un âge donné - et en-deçà de laquelle le sevrage est souhaitable. Ceci a été bien montré lorsque le sevrage était suivi d'une finition à l'herbe : ainsi McHugh \& CANNON (1959), KilleEN (1960), Wardrop et al. (1960) travaillant sur agneaux finis à l'herbe après sevrage ont montré qu'il n'y a aucun intérêt à sevrer les agneaux lorsque l'herbe n'est pas limitante, et donc que les performances des animaux (production laitière des mères et croissance des agneaux) sont, en moyenne, élevées. Plus précisément, CANNON \& BATH (1967) ont établi qu'il existe un chargement seuil en dessous duquel il est plus intéressant de laisser les agneaux sous la mère, mais en dessus duquel les agneaux doivent être sevrés. McHugH \& CANNON (1959), d'autre part, ont montré que la croissance d'agneaux issus de mères médiocres laitières augmentait après sevrage. Plus généralement, Furnival \& CoRBETT (1976) ont déterminé un poids vif critique des agneaux à six semaines, au-delà duquel il est préférable de les laisser sous la mère, mais en deçà duquel il est préférable de les sevrer.

Ces résultats entraînent des conséquences immédiates au niveau de la conduite du troupeau : après l'agnelage, celui-ci a une structure complexe, et les éleveurs herbagers constatent que les agneaux qui posent des problèmes de finition tardive sont essentiellement issus de portées multiples ou des mères les moins bonnes laitières. Une solution à ce problème serait d'effectuer un sevrage adapté au type d'agneau : précoce pour ceux qui ont une faible vitesse de croissance sous la mère, plus tardif pour les autres.

Quant à l'état d'engraissement de la carcasse, nous n'observons généralement pas de différences significatives entre les lots sevrés à 6,12 ou 16 semaines pour les différents critères d'engraissement mesurés. Ces résultats confirment ceux de SEARLE \& GRIFFITHS (1976). 
En revanche, les carcasses des agneaux sevrés présentent moins de gras péristomacal et de gras périrénal que celles des agneaux non sevrés. Ces résultats sont en accord avec ceux cités en introduction et sembleraient confirmer que le sevrage permet de réduire l'état d'engraissement d'une carcasse.

Cependant, il apparaît, chez les agneaux mâles, que le gras dorsal sous-cutané n'est pas affecté par le sevrage comme le sont les précédents critères d'engraissement.

En fait, il semblerait que le dépôt de gras sous-cutané est positivement corrélé avec la vitesse de croissance de l'animal (Murray \& Slecazek, 1976). Or, la vitesse de croissance des agneaux mâles entre la naissance et l'abattage a été de $284 \mathrm{~g} / \mathrm{j}$ contre $271 \mathrm{~g} / \mathrm{j}$ en 1981 et de $305 \mathrm{~g} / \mathrm{j}$ contre $268 \mathrm{~g} / \mathrm{j}$ en 1982 respectivement pour les sevrés et les non sevrés.

Cependant, ces différences de vitesse de croissance ont aussi été observées pour les femelles ( $272 \mathrm{~g} / \mathrm{j}$ pour les femelles sevrées et $239 \mathrm{~g} / \mathrm{j}$ pour les femelles non sevrées) et n'ont pas eu les mêmes conséquences.

Il faut toutefois rappeler que la mesure de l'épaisseur de gras dorsal est assez imprécise et que cette mesure doit être utilisée avec précaution.

Les résultats obtenus montrent une grande similitude entre deux années climatiquement différentes, élément capital dans ce type d'étude.

Dans nos conditions d'élevage, la vitesse de croissance des agneaux sous la mère, constitue un élément clé de la décision de sevrage.

Un animal à vitesse de croissance lente doit être sevré précocement puis fini en bergerie; un animal à vitesse de croissance plus rapide peut être laissé plus longtemps sous la mère et sevré uniquement lorsque les conditions deviennent défavorables au pâturage ou lonsqu'il devient pubère.

Avec un troupeau de prolificité élevée (2,16 agneaux nés, 1,43 agneaux allaités par femelle), ce type de conduite peut permettre de finir les agneaux à l'âge de 128 jours en moyenne, avec un apport de 44,4 UFV par agneau, soit 2,25 UFV de concentré par kg de carcasse produit (résultats tirés des données de l'expérience réalisée en 1982).

Reçu en octobre 1984. Accepté en juin 1986.

\section{Summary \\ Lamb production at pasture \\ I. Effect of age at weaning on the performance of lambs at pasture until weaning and then finished indoors}

This study was made to determine the interest of weaning and the age at which it should take place. Animals were subjected to the following management system : lambing and suckling indoors during the first six weeks, turnout to pasture until weaning with ad libitum concentrate supplementation and then indoors finishing.

Four treatments were tested : weaning at 6, 12 or 16 weeks old (groups 1,2,3) or absence of weaning and grazing until slaughter (control group). After weaning, each group was divided into 3 (1981) or 2 (1982) batches according to the growth rate before weaning (table 2). 
During the experiment the mean herbage allowance was high :6,9 $\mathrm{kg}$ DM per suckling ewe and per day.

Even with these very favourable conditions, the growth rate of lambs at pasture remained related to their growth rate during the first six weeks of age (table 6). By contrast, growth rate increased after weaning and did not depend anymore on that before weaning.

The lowest concentrate intake was obtained with weaning at 16 weeks in lambs exhibiting a rapid growth rate during suckling and at 12 weeks in lambs with a low growth rate (tables 7 and 8 ).

These results suggest that lambs growing slowly during suckling should be weaned early, whereas lambs growing more rapidly should be weaned later. This strategy would result in concentrate sparing and ealier lamb finishing (table 5). Results were similar for both years.

Key words : Age at weaning, weaning, grazing lambs, lambs.

\section{Références bibliographiques}

Cannon D.J., Bath J.G., 1967. Prime lamb production : the influence of stocking rate on response to early weaning. Aust. J. Exp. Husb., 7, 11-16.

Fontanilas R., 1971. La production d'agneaux d'herbe : exploitation intensive des fourrages verts et complémentation. Journées CETA, Etude $\mathrm{n}^{\circ} 1786$.

Furnival E.P., Corbett J.L., 1976. Early weaning of grazing sheep - I. Growth of lambs. Aust. J. Exp. Husb., 16, 149-155.

GibB M.J., Treacher T.T., 1980. The effect of ewe body condition at lambing on the performance of ewes and their lambs at pasture. J. Agric. Sci. Camb., 95, 631-640.

JoNES R.J., SANLAND R.L., 1974. The relation between animal gain and stocking rate Derivation of the relation from the results of grazing trials. J. Agric. Sci. Camb., 83 (2), 335-342.

KILLEEN I.D., 1960. Rearing of young sheep with special reference to early weaning. Wool Technology and Sheep Breeding, 7 (1), 33.

MacHugh J.F., Cannon D.J., 1959. Early weaning of lambs. J. Agric., Victoria, 57, 223.

MaItre C., 1980. Evolution technico-économique d'un troupeau ovin de plein air. Pâtre, janvier 1980, 23-30.

Maitre C., 1982. La finition des agneaux d'herbage. Pâtre, juin-juillet 1982, 42-46.

Martin J.L., 1982. La finition des agneaux de plein air : différentes possibilités. La France Agricole, septembre 1982, 50-52.

Mitchell, R.M., JAGUSH K.T., 1972. Energy balance studies with weaned lambs. I. Utilization of metabolisable energy of lucerne by lambs weaned at 5 weeks of age. N.Z. J. Agric. Res., 15, 788-794.

Murray D.M., Slecazek O., 1976. Growth rate and its effect on empty body weight, carcass weight and dissected carcass composition of sheep. J. Agric. Sci. Camb., 87, 171-179.

Orskov E.R., Fraser C., Gill J.C., Corse E.L., 1971. The effect in an intensive production system of type of cereal and time of weaning on the performance of lambs. Anim. Prod., 13, 485-492.

Penning P.D., Gibi M.J., 1979. The effect of milk intake on intake of cut and grazed herbage by lambs. Anim. Prod., 29, 53-67.

Robelin J., Theriez M., Arnal M., Perrara M., 1977. Evolution de la composition chimique des jeunes agneaux mâles jusqu'à l'âge de 16 semaines. Ann. Zootech., 26 (1), 69-81. 
RUTTER W., 1970. A comparison of the performance of suckled lambs with lambs weaned at seven weeks of age and either grazed or given concentrates. Anim. Prod., 12, 543-549.

Searle T.W., Griffiths D.A., 1976. The body composition of growing sheep during milk feeding and the effect on composition of weaning at various body weights. J. Agric. Sci. Camb., 86, 483-493.

Theriez M., 1984. The young lamb. Livestock Feeds and Feeding, D.C. Church Ed., O.B. Books Corvallis, Chap. 18, 339-353.

U.C.A.A.B., 1979. Complémentation des agneaux à l'herbe. Bulletin Technique de l'U.C.A.A.B., $\mathrm{n}^{\circ}$ 3, 19-26.

VAN QuackebeKe E., 1983. Comportement des agneaux d'herbe - Problèmes de finition. VIII $^{\mathrm{es}}$ Journées de la Recherche Ovine et Caprine, I.N.R.A. - I.T.O.V.I.C. - Exploitations des fourrages verts par les ovins et les caprins, 153-186.

VAN QUACKeBEKE E., 1976. Rentabilités et perspectives : l'engraissement des agneaux à l'herbe ou en bergerie. Revue de l'Elevage, numéro hors série, 59-65.

VilletTe Y., THeriez M., 1981. Influence du poids à la naissance sur les performances d'agneaux de boucherie. I. Niveau d'ingestion et croissance. Ann. Zootech., 30, 151-168.

Wardrop I.D., Tribe D.E., COOMBE J.B., 1960. An experimental study of the early weaning of lambs. J. Agric. Sci., 55 (1), 133-136.

Young N.E., NewToN J.E., 1975. A comparison between rotational grazing and set stocking with ewes and lambs at three stocking rates.

Anim. Prod., 21, 303-311. 